\title{
Acknowledgment to Reviewers of Journal of Imaging in 2020
}

\section{Journal of Imaging Editorial Office}

MDPI AG, St. Alban-Anlage 66, 4052 Basel, Switzerland

Citation: Journal of Imaging Editorial Office. Acknowledgment to Reviewers of Journal of Imaging in 2020. J. Imaging 2021, 7, 14. https:// doi.org/10.3390/jimaging7020014

Published: 25 January 2021

Publisher's Note: MDPI stays neutral with regard to jurisdictional

claims in published maps and institutional affiliations.

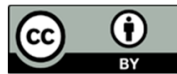

Copyright: () 2021 by the author. Licensee MDPI, Basel, Switzerland. This article is an open access article distributed under the terms and conditions of the Creative Commons Attribution (CC BY) license (http://creativecommons.org/licenses/by/4.0/).

Peer review is the driving force of journal development, and reviewers are gatekeepers who ensure that Journal of Imaging maintains its standards for the high quality of its published papers. Thanks to the cooperation of our reviewers, in 2020, the median time to first decision was 20.5 days and the median time to publication was 50 days. The editors would like to express their sincere gratitude to the following reviewers for their precious time and dedication, regardless of whether the papers were finally published:

Abdellatif, Mohamed

Abdel-Nasser, Mohamed

Abel, Andrew

Abelha Mota, Guilherme Lucio

Abir, Muhammad

Aghaei, Maya

Ahmed, Wael

Aldea, Emanuel

Alidoost, Fatemeh

Aliotta, Francesco

Almeida, Marcos

Al-qershi, Osamah

Alzubaidi, Laith

Ambroziak, Andrzej

Amini, Marzieh

Amiri, Sam

Ampeliotis, Dimitris

Anagnostopoulos, Ioannis

Andreić, Željko

Antunes, Vanessa

Arguelles-Cruz, Amadeo J.

Awad, Ali Said

Azetsu, Tadahiro

Bacanin, Nebojsa

Bae, Sung-Ho

Baek, Seungryul

Bahmanyar, Reza

Balado Frías, Jesús

Bamatraf, Saeed M.

Barjaktarović, Marko Č.

Barker, Michael Kenneth

Barnes, Anna

Batchuluun, Ganbayar

Becek, Kazimierz

Beecks, Christian

Begušić, Dinko
Belaid, Abdel

Bengtsson, Ewert

Berg, Carl Fredrik

Bernstein, Alexander V.

Bevacqua, Martina Teresa

Bevitt, Joseph

Bianconi, Francesco

Błaszczak-Bąk, Wioleta

Bobkowska, Katarzyna

Bobulski, Janusz

Bobyr, Maxim

Bock, Joel R.

Bogunović, Hrvoje

Bollmann, Steffen

Bordoy, Joan

Bouhrara, Mustapha

Bowman, Adrian

Brehar, Raluca

Brudfors, Mikael

Buonamici, Francesco

Calvo-Zaragoza, Jorge

Cândido Carvalho, Kátia

Canetta, Elisabetta

Carbon, Claus-Christian

Caron, Guillaume

Castejón-Limas, Manuel

Castellanos Regalado, Francisco José

Cebollada, Sergio

Celona, Luigi

Cernadas, Eva

Chan, Kwok-Ping

Chang, Kuo-Jen

Chauhan, Vedang Dilipkumar

Chaves, Deisy

Chen, Hung-Shing

Chen, Siming 
Chen, Yu-Jen

Chetouani, Aladine

Chicchi Giglioli, Irene Alice

Choi, Yongho

Chromy, Adam

Chuang, Tzu-Yi

Chuirazzi, William

Cintra, Renato J.

Ciuonzo, Domenico

Cizelj, Leon

Coccia, Mario

Cocianu, Cătălina-Lucia

Conceicao, Raquel

Coronel, Anibal

Corti, Martina

Cosma, Georgina

Craft, Aaron E

Cruz-Hernández, César

Cuocolo, Renato

Czajkowska, Joanna

Da Silva Soares, Anderson

Dalitz, Christoph

Dang-Nguyen, Duc-Tien

Daoud, Bilel

Dawson, Jeremy

De Marco, Matteo

De Mingo López, Luis Fernando

De Paiva, Anselmo Cardoso

De Reijke, Theo M.

Delibasis, Konstantinos K.

Demidenko, Eugene

Demidova, Liliya

Deng, Tiantai

Deniziak, Stanisław

Di, Zichao

Diaz-Cortes, Margarita

Diem, Markus

Diez, Yago

Dijkstra, Jouke

Dingliana, John

Diraco, Giovanni

Dobrez, Livio

Dogaru, Radu

Domínguez-Morales, Juan P.

Dorizzi, Bernadette

Dorota, Majorkowska-Mech

Doutch, James

Doutsi, Effrosyni

Dresp-Langley, Birgitta

El Adoui, Mohammed

El Yacoubi, Mounim A.
Elbahnasawy, Magdy

Elmogy, Mohammed

Elwahab, Sami Abd

Estomba, Carlos Miguel Chiesa

Fang, Jian

Fanti, Alessandro

Farmer, Michael

Feng, Hsuan-Ming

Fernandes, Henrique C.

Fernández-Caballero, Antonio

Ferrari, Claudio

Ferreira, Artur

Ferreira, Fernando

Festa, Giulia

Feuerhake, Friedrich

Finkbeiner, Steven

Fiorentino, Maria Chiara

Flusser, Jan

Fonseca-Pinto, Rui

Forzani, Erica

Fox, Richard

Freitas, Pedro Garcia

Fujiwara, Takeshi

Gade, Rikke

Gaffar, Ashraf

Gallego, Antonio-Javier

Gao, Zhan

Gasparini, Francesca

Gatto, Bernardo B.

Gavrilov, Dmitry

Gavrilova, Marina

Gavrovska, Ana

Gawronek, Pelagia

Gebremariam, Kidane Fanta

Gerard, Sarah

Ghidoni, Stefano

Ghosal, Sambuddha

Giannakeas, Nikolaos

Gibbs, John

Giovanacci, David

Golnabi, Amir H.

Grosse, Mirco

Guaragnella, Cataldo

Guevara-Lopez, Miguel Angel

Guidotti, Patrick

Günther, Manuel

Hakoyama, Tomoyuki

Hamel, Lutz

Harada, Masahide

Harizanov, Stanislav

Hautière, Nicolas 
Havig, Paul

Havlicek, Joseph P.

Heidari, Morteza

Hellbourg, Gregory

Ho, Edmond S. L.

Horgan, Graham

Hossain, Belayat

Hosseini, Seyed M. H.

Hosu, Vlad

Hotz, Ingrid

Hożyń, Stanisław

Hsu, Chih-Chung

Huang, Shaoguang

Huo, Yumei

Hussain, Zahir

Hwang, Yin-Tsung

Hwang, Youngbae

Ibarra-Manzano, Oscar

Imani, Mahdi

Ingold, Rolf

Intzes, Ioannis

Inzerillo, Laura

Ionescu, Cringu

Ipek, Özlem

Irianto, Jerome

Irrgang, Christopher

Ivashchenko, Olena

Jafri, Rabia

Jansing, David

Jantzen, McNeel G.

Jaworska, Tatiana

Jeon, Byunghwan

Jester, James

Jochmanova, Ivana

Juan, Guillermo

Jubery, Talukder Z.

Kaestner, Anders

Kamaleswaran, Rishikesan

Kampa, Adrian

Karakatič, Sašo

Kardjilov, Nikolay

Karimov, Timur

Katkovnik, Vladimir

Khademi, April

Khan, Rizwan Ahmed

Kichanov, Sergey

Kim, Wonjun

Kim, Yoon-Chul

Kirchgessner, Norbert

Klemeš, Jiří Jaromír

Kline, Timothy L.
Knyaz, Vladimir

Kockelmann, Winfried

Koike, Takahiko

Kojima, Tetsuya

Kolb, Andreas

Kopczyński, Eryk

Korecki, Pawel

Kose, Utku

Kothapalli, Sri-Rajasekhar (Raj)

Koutsoudis, Anestis

Kramer, Tobias

Kranjčić, Nikola

Krejcar, Ondrej

Krivoshein, A.

Kumar, Shitij

Kuriki, Ichiro

Kwon, Sunghoon

Ladevèze, Sandrine

Lagares, Alfonso

LaManna, Jacob

Lanteri, Luca

Lara-Cueva, Román Alcides

Lavoué, Guillaume

Lazzaretti, André Eugênio

Le, Hoang Ngan

Lèbre, Marie Ange

Lee, Hyunkwang

Lee, Jinseok

Lee, Kwonmoo

Lee, Sang Jun

Lee, Sanghoon

Lee, Seunggyu

Lehmann, Eberhard

Leishman, Robert C.

Li, Haoliang

Li, Hongliang

Li, Yung-Hui

Lin, Cheng-Hung

Lin, Huei-Yung

Lin, Shinfeng

Lin, Xiao

Litany, Or

Liu, Bin

Liu, Sheng-Chi

Liu, Shengheng

Liu, Suxing

Livieris, Ioannis E.

López Granado, Otoniel Mario

Lukinac, Jasmina

Lukomski, Michal

Lukoševičius, Saulius 
Lukosi, Eric

Luqman, Muhammad Muzzamil

Mabrouk, Rostom

Machikhin, Alexander

Magalhães, Ricardo

Makek, Mihael

Mandal, Subhamoy

Marasović, Tea

Marcato Júnior, José

Markelin, Lauri

Marone, Federica

Martin, Iain

Martínez Torres, Javier

Martins, Diana

Mascarenhas, Nelson D. A.

Masiero, Andrea

Mastrotheodoros, Georgios P.

Matic, Tomislav

Matsubara, Edson Takashi

Mazurek, Przemysław

McKenna, Stephen J.

Meerwald-Stadler, Peter

Meiburger, Kristen M.

Mercier, Gregoire

Merrill, Frank

Mielewczik, Michael

Migallón, Héctor

Mihelič, Jurij

Milicevic, Mario

Miller, Alexander

Miller, Boris

Milosavljević, Aleksandar

Minaee, Shervin

Mishra, Deependra

Mocanu, Irina

Moccia, Sara

Moldovanu, Simona

Monno, Yusuke

Montel, Denise

Montisci, Augusto

Moon, Hyeonjoon

Morgado, António Miguel

Morgano, Manuel

Morillas Gómez, Samuel

Morris, Christopher

Mousas, Christos

Muhammad Arslan, Usman

Müller, Ulrich

Munaiz, Eduardo D.

Muñoz, Adolfo

Myers, Kenneth A.
Nagai, Takeharu

Naik, Ganesh R.

Nakagawa, Masaki

Navarro, Nicolas

Nguyen, Van Phuc

Nicholson, Kerry Cawse

Nicolas, Stéphane

Nocerino, Erica

Norton, Kerri-Ann

Nuutinen, Mikko

O'Loughlin, Declan

O'Regan, Declan

Oikawa, Kenichi

Okarma, Krzysztof

Olaru, Mihaela

Oniga, Florin

Paek, Sung Wook

Paeng, Dong-Guk

Pagnozzi, Alex

Pakula, Anna

Panchapakesan, Balaji

Pandey, Prem Chandra

Paquet, Thierry

Park, Ji Hwan

Parkinson, Dilworth

Parnell, Steven

Pasquini, Cecilia

Pastena, Massimiliano

Pastorino, Matteo

Paternain, Daniel

Pelc, Mariusz

Pelt, Daniël M.

Peng, Yan-Tsung

Peng, Yifan

Pepe, Francesco V.

Peter, Pascal

Petrellis, Nikos

Pibernik, Jesenka

Pino, Esteban J.

Pires, Ivan Miguel Serrano

Piva, Alessandro

Plech, Anton

Posada, Jorge

Pouli, Tania

Pouliakis, Abraham

Prasad, Dilip K.

Puppe, Frank

Putzu, Lorenzo

Qi, Guanqiu

Qi, Zheng

Qian, Chunqi 
Rabaev, Irina

Radvan, Roxana

Rahman, Mahmudur

Rao, Kamisetty R.

Rao, Muzaffar

Reber, Jonas

Reeves, Adam

Refice, Alberto

Ren, Wuwei

Ren, Yuzhuo

Rengarajan, Amirtharajan

Renson, Ludovic

Reynolds, Hayley

Ribas, Salvador

Richter, Rudolf

Riebe, Danielle

Righi, Marco

Rodriguez, Jeffrey J.

Roger, Jean-Claude

Roig, Ignacio Bosch

Romanuke, Vadim

Romeo, Stefania

Roșca, Daniela

Roy, Sudipta

Ruijsink, Bram

Rusu, Mirabela

Ruszczak, Bogdan

Ryu, Kanghyun

Saabni, Raid

Safonov, Ilia

Saha, Sudipan

Sajjanhar, Atul

Salido Tercero, Jesús

Sanchez, Victor

Sanli, Fusun

Santos, Florentino

Sawall, Stefan

Saxena, Shefali

Schaff, Florian

Schillinger, Burkhard

Schläffer, Martin

Schoenhagen, Paul

Schregle, Roland

Sebastião, Helder

Selci, Stefano

Semeniuta, Oleksandr

Sequeira, Ana

Serra-Sagristà, Joan

Sfarra, Stefano

Sgalaberna, Davide

Shafiq, Omair
Shah, Jainil

Sharma, Ashutosh

Sharma, Sonisa

Shevkunov, Igor

Siemiatkowska, Barbara

Simon-Chane, Camille

Singh, Saurabh

Skopal, Tomáš

Smith, Rhodri

Sobieranski, Antonio Carlos

Sochen, Nir

Solano-Correa, Yady Tatiana

Soltaninejad, Mohammadreza

Song, Youngseok

Sparavigna, Amelia Carolina

Srinivasan, Ravi

Stavropoulou, Eleni

St-Charles, Pierre-Luc

Stoean, Catalin

Stojanovic, Branka

Stommel, Martin

Strobl, Markus

Strohmaier, Walter L.

Strzelecki, Michal

Su, Hang

Su, Yuhua

Sujit, Sheeba Jenifer

Sulavko, Alexey

Sun, Ke

Suwanwiwat, Hemmaphan

Szczepanowska, Hanna

Tallents, Greg J.

Tang, Zhaohui

Tangherloni, Andrea

Tan-Showyin, Jackie

Tarasov, Andrei

Tarasov, Dmitry

Tavakolian, Pantea

Teixeira, Jose

Teng, Liong Sze

Theodorakopoulos, Ilias

Thomsen, Knud

Tian, Gui Yun

Tinelli, Andrea

Tinevez, Jean-Yves

Tonacci, Alessandro

Trăscău, Mihai

Treimer, Wolfgang

Tremsin, Anton S.

Trtik, Pavel

Trusiak, Maciej 
Tzilas, Vassilios

Uddin, Jia

Ulacha, Grzegorz

Ullah, Mohib

Ullah, Zahid

Valsesia, Diego

Valtchinov, Vladimir I.

Van Eijck, Lambert

Vasquez, Jorge A. Tobon

Vassilakis, Emmanuel

Vaughan, Christopher L.

Venkataraman, Rajesh

Vijayaraghavan, Gopal R.

Vocaturo, Eugenio

Wang, Haifeng

Wang, Qian

Wang, Shuihua

Wang, Shuo

Wang, Shuohong

Watkins, Eric

Watson, Des

Werman, Michael

Wick, Christoph

Widerska-Chadaj, Zaneta S

Williams, Jeremiah
Wollmann, Philipp

Wrzesiński, Grzegorz

Wysocki, Marian

Xiao, Ran

$\mathrm{Xu}$, Xiaowen

$\mathrm{Xu}$, Yaping

Yang, Jie

Yang, Lee-Xieng

Yao, Aiping

Yasrab, Robail

Yoo, Jung Sun

Yoon, Hyungchul

Youssof Zadeh, Vahab

Yu, Ting-To

Yuan, Yixuan

Zambrano-Martinez, Jorge Luis

Zamfir, Rares Halbac-Cotoara

Zeng, Kai

Zhang, Lei

Zhang, Ming

Zhao, Mingjun

Zhou, Jun

Zolliker, Peter

Zubrzycki, Jarosław 